\title{
A Novel Technique for Enhancing Color of Undersea Deblurred Imagery
}

\author{
Chrispin Jiji*, Nagaraj Ramrao
}

Department of Electronics and Communication, The Oxford College of Engineering, Oxford Institutions, Bangalore, India

\begin{tabular}{l} 
A R T I C L E I N F O \\
\hline Article history: \\
Received: 18 September, 2018 \\
Accepted: 23 October, 2018 \\
Online: 01 November, 2018
\end{tabular}

Keywords:

Underwater

Image Deblurring

Image Enhancement

\begin{abstract}
A B S T R A C T
Exploring the ocean underneath has always been an area of great scientific and environmental concern. However, the study of underwater environment was very difficult due to the extreme conditions. Undersea descriptions undergo severe distortion attributed to absorptive as well as scattering properties. Absorption substantially removes illumination, whereas a ray of light redirected in several path when it interacts by substance. Because of these, undersea descriptions encompass blur as well as color loss. In this paper we suggested an effective technique namely, a turbidity removal method for deblurring the image. If the deblurred image has a lighting problem, we make use of a color-correction method to find the clear image. Our substantial qualitative and quantitative assessment expose that the proposed algorithm progress the excellence as well as lessen color distortion loyally, also improves the state-of-the-art undersea technique.
\end{abstract}

\section{Introduction}

Images captured in undersea has plays a vital basis of interest within various branches of technical and systematic explores [1], such as examining underneath infrastructures [2] as well as cables [3], detecting manmade objects [4], managing undersea vehicle [5], marine biology investigate [6], and archaeology [7]. Apart of normal descriptions, undersea descriptions undergo reduced visibility ensuing attenuation of the propagated illumination, mainly owing to absorption along with scattering effect. In this paper, we use image processing acting extensive interest over earlier years due to its challenging nature and its importance for the surroundings. Improving undersea scene excellence separates the problem into image restoration and image Enhancement

Visibility in undersea imagery is usually blurry, but having large number of particles underneath cause's cloudiness or more haziness, called turbidity, which causes blur in undersea imagery. To remove any blur, we usually use restoration problem with estimated or known PSF matrix.

$$
b=h * d+n
$$

where $d$ represents deblurred representation, $h$ denotes PSF kernel, and $b$ denotes turbid representation. The main challenge is estimating blur kernel [8-12]. The blur kernel cannot be estimated directly as it varies depending on blur itself. Existing system uses some prior to estimate point spread function (PSF) for restoration of undersea blurred imagery.

${ }^{*}$ A. Chrispin Jiji, 8951627124 \& chrispinjij@gmail.com
If the deblurred image has any difficulty in lighting, we use image enhancement to enhance the picture excellence. The difficulty during lighting owing towards absorption substantially reduces illumination, practically, in undersea images shown in Figure 1. The objects by remoteness more than $10 \mathrm{~m}$ are about imperceptible, and colors go down by the deepness of water.

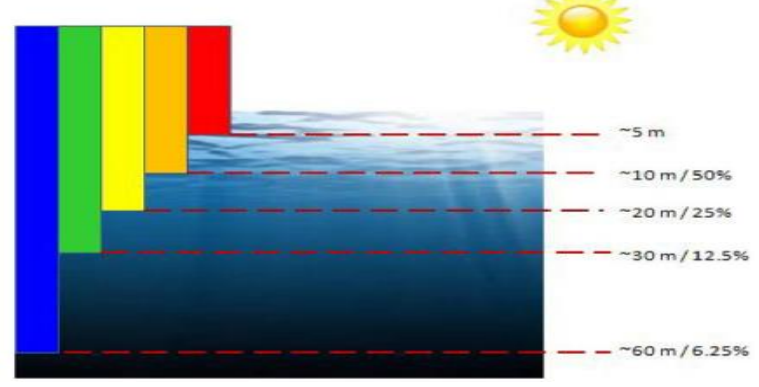

Figure 1: Dissimilar illumination weakened by dissimilar charge

Define abbreviations and acronyms the first time they are used in the text, even after they have been defined in the abstract. Do not use abbreviations in the title or heads unless they are unavoidable. In general, red illuminations vanish by deepness of $5 \mathrm{~m}$, after that brown followed by yellow radiance, lastly green as well as blue illumination vanish by deepness with $30 \mathrm{~m}$ as well as $60 \mathrm{~m}$. Thus undersea imagery is subject to blue-green color which changes the picture excellence. As a result, we used color adjustment scheme to compensate light condition. There have been several attempts for restoration as well as enhancement, since degraded picture 
outcomes the understanding of multiplicative by means of additive process [13]. Conventional enhancement methods namely gamma correction, histogram equalization strongly restricted for such a task. This difficulty deal with modified attainment approach via various imagery [14], specific hardware [15] or polarization filters [16]. Even though their important attainment, these approach undergo numeral concern that decrease their convenient applicability.

As shown in Figure 2, our technique uses two stages namely image restoration stage for deblurring and enhancement stage for enhancing its excellence. In this paper we suggest an adaptive sparse domain selection (ASDS) system to restore the image. By training compact sub-dictionaries from high quality example image cluster the window. Since each cluster employ several windows by related prototype, compact sub-dictionary learns for every cluster. Particularly, for simplicity we use principal component analysis (PCA) technique towards learning subdictionaries. The most excellent sub-dictionary that is mainly applicable to given window is chosen, because the given window is better represent sub-dictionary is accurately reconstruct entire image. Besides sparsity regularization, other terms as well initiate for improving its performance. Later use autoregressive (AR) models, pre-learned from training dataset characterizing confined structures. For every confined window, we choose the AR model. On the other hand, considering fact that there are often several repetitive image structures in an image, we introduce a non-local (NL) self-similarity constraint served as another term, helpful for preserving boundary sharpness and restrain noise. After introducing ASDS using two constraints (AR \& NL) into IR structure, we present a weighted Gray Edge method for solving lighting problem. Substantial experimentation on image deblurring and enhancement show that the projected approach effectively reconstructs picture details along with excellence, outperforming different state-of-the-art IR with IE methods in terms of both excellence metrics in addition to ocular insight.

The paper is planned as follows. Section 2 provides concise outline about earlier art. Section 3 signifies our restoration approach, about turbidity removal method especially used for undersea descriptions. Section 4 describes novel enhancement approach, mainly to improve its excellence. Section 5 presents comparative qualitative and quantitative estimation of undersea system and Section 6 provides closing comments.

\section{Previous Art}

This part reviews main advances to deblur or else improve imagery confined undersea. In computer vision, methods to handle ambiguity is roughly on some knowledge or assumptions known beforehand, i.e., priors. The priors impose extra constraints/dependency among the unknown variables. In the following, we review the previous turbidity removal methods. We do not discuss the technical details among those methods. Instead, we concern about the extra constraints. All methods reformulate in a same framework expressed indifferent forms in the original works. Single image turbidity removal methods have to rely on some priors.

The prior is statistical/physical properties, heuristic assumptions, simplifications, along with application-based rules. The blur imaging model in (1), discrepancy between some equations and unknown, the prior expected to introduce at least one constraint for each pixel. The challenge of recovering $d$ from $b$ is under-constrained. To make it solvable, extra knowledge has been built-into the restoration method. The former understanding is often built-in with a regularization term, principal to the later energy minimization problem:

$$
\hat{d}=\arg \min \left\{\|b-H d\|_{2}^{2}+\lambda J(d)\right\}
$$

where $\lambda$ denotes Lagrangian multiplier matching the exchange among former term $J(d)$ along with likelihood $\|b-H d\|_{2}^{2}$. The former term $J(d)$ act as a main part in the restoration method. Sparsity based IR process guess that the natural representation is sparse in few fields. Sparse representation lately has paying attention to investigators for resolving complications including deblurring, denoising, super resolution. In [17], the ARM prototypes are in the neighborhood calculated commencing an originally expected image besides bring about much better-quality for TV prior trendy reforming boundary associations. Now, we resolve to suggest knowledge established using adaptive prior, where the AR prototypes remain knowledgeable commencing great feature of training imageries, towards raising AR forming accuracy. The approach presents exact modest: the blotches that make sure alike forms can exist spatially distant, besides gather the entire appearance. In [18-20], the NLM prior combined through the sparse domain, as of alike appearance blotches be concurrently implied towards the strength of converse reestablishment.

Image enhancement based methods are not required to solve the physical form, but rather directly enhance its contrast as well as improving its excellence from human visual perception. Within undersea, color is extremely related by deepness, and a significant difficulty is green-bluish form desires to resolve. Since as the illumination go through undersea, reduction process affects wavelength spectrum, thus affecting the gray level along with appearance of colored surface. Existing white balancing process is a key to our domain. Next, we briefly change those techniques and give details for our novel approach projected by undersea imagery. Generally a scheme formulates exact guess to estimate color, in addition to meet color constancy via color channel will standardize the radiance. Projected scheme use a statement that majority of patch show off extremely small gray level in at-least single color channel that openly about blur mass and progress vivid colors. It cannot sufficiently deal with color deformation and complex arrangement. The deblurred imagery will attribute color swing with artifact effect. The Grey World system [21] believe that the averages of the three color constituent, illuminate during impartial radiance basis further acquire poorer color patch. The Max-RGB [21] resting on the RGB color. Shades-of-Grey [22] use Minkowski norm-p is achromatic. The Grey Edge method [23] employs items boundary data towards adjusting color fidelity; it assumes that normal derivative of color mechanism is achromatic. Every color provides pre procedure by Gaussian filter is typically 1-2 toward determining the statement. It might worsen the performance. Weighted Grey Edge method [24] use boundary data of variety of substance, intended for instance, shadow and mechanism. This paper is an extended work initially presented during ICPCSI [25]. Also the journal paper intended novel technique for enhancing undersea imagery. Revised result much 


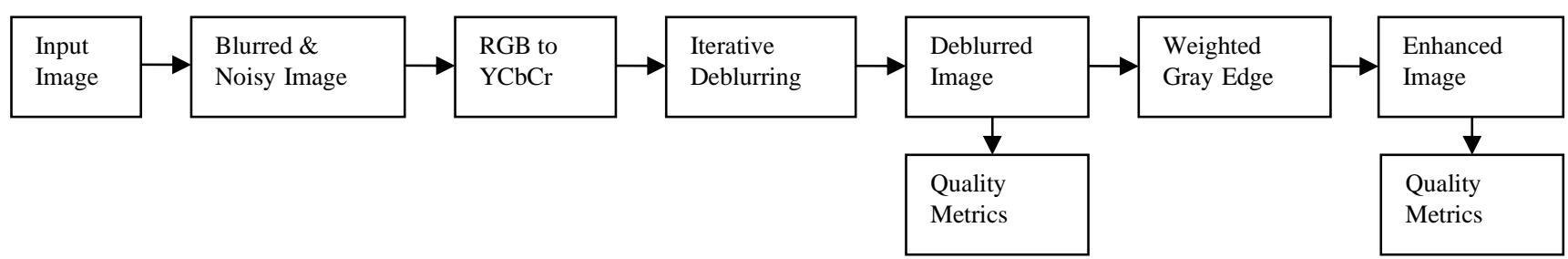

Figure 2: Block diagram of Proposed Method

improves the lighting problem in undersea imagery. Enhancement based methods not required to solve physical form of degraded image, but rather directly enhance contrast and improve image quality from human visual perception.

\section{Underwater image restoration for turbidity removal method}

Underwater turbidity removal is very challenging with essential part of picture suffers from turbidity. Several algorithm deals with turbidity elimination process recognized as Image deconvolution (ID). To get back the undersea deblurred picture, we used [26-27]. Here we adaptively study compacted sub-dictionaries to every confined patch. Let $d_{m} \in R^{n}$ denote the image block dimensions $V_{\mathrm{n}} \times \sqrt{\mathrm{n}}_{\mathrm{n}}$ attained from deblurred picture, we can describe $d_{m}=R_{m} D, m=1,2, \ldots \ldots . . N$ wherever $R_{m}$ denotes matrix from $d_{m}$. Rendering towards sparse prior $d_{m}$ can be signified by means of outmoded dictionary, namely $d_{m}=\phi_{k_{m}} \alpha_{m}$, thus $D$ can be conveyed by way of

$$
D=\left(\sum_{m=1}^{N} R_{m}^{T} R_{m}\right)^{-1} \sum_{m=1}^{N} R_{m}^{T} \phi \alpha_{m}
$$

For convenience, we define:

$$
D=\phi o \alpha=\left\{\sum_{m=1}^{N} R_{m}^{T} R_{m}\right\}^{-1} \sum_{m=1}^{N} R_{m}^{T} \phi \alpha_{m}
$$

Now, the primary restored image denoted as $D^{(k)}$ constantly drop some information in the input image and comprises some artifacts. In order to further appropriate for human visual scheme, we update the restored image in every iteration.

For each patch we adaptively choose the sub-dictionary based on least distance specified through

$$
k_{m}=\left\|d_{m}^{h}-\mu_{k}\right\|_{2}^{2}
$$

\subsection{Adaptively Reweighted Sparsity Regularization}

Existing work uses $\lambda=1$ for whole patches. In our work $\lambda$ differs from patch to patch to expand the excellence of restored image. The reweighted sparsity regularization is specified in place of

$$
\hat{\alpha}=\left\{\|b-H \phi o \alpha\|_{2}^{2}+\sum_{m=1}^{N} \sum_{n=1}^{o} \lambda_{m, n}\left|\alpha_{m, n}\right|\right\}
$$

Then weight $\lambda_{m n}$ stands as

$$
\lambda_{m, n}=\frac{2 \sqrt{2} \sigma_{n 1}^{2}}{\sigma_{m, n}+\varepsilon}
$$

Reweighted procedure is as follows:

Step $1 \quad$ Fix $\ell=0$ as well as $\lambda_{n}^{l}=1$

Step 2 Resolve the subjective $l_{1}$ minimization problematic

$$
d^{(l)}=\arg \min \left\|\lambda_{n}^{l} \phi^{T} d\right\|_{1} \text { Focused towards } d=\phi \alpha^{(l)}
$$

Step 3 Set $\alpha^{(l)}=\phi^{T} d^{(l)}$ and describe

$$
\alpha_{n}^{(l+1)}=\frac{2 \sqrt{2} \sigma_{n 1}^{2}}{\sigma_{m, n}+\varepsilon}
$$

Step 4 Proceed until $l$ reaches $l_{\max }$ Else, increase $l$ and drive towards step 2

\subsection{Auto Regressive model}

The ASDS progress extensively by the usefulness of sparse modeling and later outcome image deblurring. To further get better excellence of deblurred imagery, we established autoregressive (AR) models to formalize the image confined smoothness. For each high pass filtered patch adaptively choice AR dictionary by means of calculating the Euclidean distance. For each present pel we make sure four neighbouring pels and its weights are specified as;

$$
w_{m, n}=\frac{1 /\left(\left\|d_{m}^{h}-\mu\right\|_{2}^{2}+\varepsilon\right)}{\sum 1 /\left(\left\|d_{m}^{h}-\mu\right\|_{2}^{2}+\varepsilon\right)}
$$

Therefore, the weighted sum of entire adjacent pels expressed as

$$
\chi_{m}=\sum w_{m n} a_{k_{m}}
$$

Assemble the exceeding matrix into $3 \times 3$ window we resolve the value of local geometry model. Let $d_{m}$ remains the center pel, and $\chi_{m}$ stays the vector comprising of the adjacent pels which is adjacent to middle pel $d_{m}$, now the best middle pel value would lessen $\left\|d_{m}-a_{k_{m}}^{T} \chi_{m}\right\|_{2}^{2}$.For the ease of expression we write $\sum_{d_{i} \in d}\left\|d_{m}-a_{k_{m}}^{T} \chi_{m}\right\|_{2}^{2}$ as $\|(I-A) \phi o \alpha\|_{2}^{2}$. By integrating this restraint, the restoration equation as follows 


$$
\hat{\alpha}=\left\{\|b-H \phi o \alpha\|_{2}^{2}+\sum_{m=1}^{N} \sum_{n=1}^{o} \lambda_{m, n}\left|\alpha_{m, n}\right|+\gamma\|(I-A) \phi o \alpha\|_{2}^{2}\right\}
$$

where $\gamma$ represents a constant balance the role of AR formalization term.

\subsection{Nonlocal Self Similarity model}

The AR model utilizes the confined information in every patch. On the other hand, there are often numerous cyclical prototypes all through the representation. Thus non-local redundancy mainly improves the excellence of imagery. Along with AR models an additional term called non-local similarity added into the IR structure. The source of global mean filtering is very simple, aimed at current image block; we catch numerous alike blocks to restrain it. Aimed at each image block $d_{m}$, we discover all image blocks alike to it in the complete image.Take on $d_{m}^{s}$ is one of blocks similar to $d_{m}$, then $e_{m}^{s}=\left\|d_{m}^{s}-d_{m}\right\|_{2}^{2}$ would be small enough. If we estimate the summation of entire midpoint pels voguish these alike blocks, besides effects would satisfy $d_{m} \approx \sum_{s=1}^{L} b_{m}^{s} x_{m}^{s}$ where $b_{m}^{s}$ denotes the weight owed to $d_{m}^{s}$.

Apparently, the more two blocks are alike; the bigger weight would be assigned. Thus, we can define the expression for weight calculation as $b_{m}^{s}=\exp \left(-e_{m}^{s} / h\right) / \sum \exp \left(-e_{m}^{s} / h\right)$ where $h$ stays a constant directing the window shape. The global model in an image can be estimated such as

$$
\sum_{m=1}^{N}\left\|d_{m}-\sum_{s=1}^{L} b_{m}^{s} d_{m}^{s}\right\|_{2}^{2}
$$

Mathematically in form of $\|(I-B) \phi \alpha\|_{2}^{2}$. Besides incorporate equally the AR regularization and the NLSS based sparse depiction in Eq. (12), thus solves the problem using AR to regularize image confined smoothness with NLSS towards utilizing the image Non-Local redundancies as given as

$\hat{\alpha}=\left\{\|b-H \phi o \alpha\|_{2}^{2}+\sum_{m=1}^{N} \sum_{n=1}^{o} \lambda_{m, n}\left|\alpha_{m, n}\right|+\gamma\|(I-A 1) \phi o \alpha\|_{2}^{2}+\eta\|(I-A 2) \phi o \alpha\|_{2}^{2}\right\}$

Meanwhile $\eta$ panels the stability among local adaptation plus nonlocal strength.

The projected technique advances the existing schemes into excellence metrics despite undersea picture is crucial to recover color. In next section, we therefore propose color adjustment approach, relying on weighted grey model. As depicted within Figure 2, our approach adopts a novel strategy, for compensating color cast so as to enhance detail scene.

\section{Underwater image enhancement of deblurred image}

The deblurred image is subject to global enhancement with no prior required. This is necessary to lessen the architects due to deblurring, and for enhancement of color information otherwise degraded in the underwater image formation process. Different depth levels allow different colors to distort. Thus the faded as well as non-uniform color distribution will describe the undersea representation. Mauricio [28, 29] proposed an algorithm to readily pre-process the underwater imaging using homomorphic filtering, wavelet de-noising anisotropic filtering, and RGB color channel equalization to enhance color. The technique is usual and need no prior or constraint change. Another such scheme uses distribution of the histogram to make the bins change according to color density. In the proposed work, we use a weighted grey edge scheme to improve the color constancy.

\subsection{Color adjustment section}

Color adjustment is crucial in undersea, we apply our system towards deblurred imagery thus improving the picture look by means of neglecting redundant color casts caused by diverse radiance. To obtain our output we achieve a gamma correction method which endeavours to correct global dissimilarity; hence progress color constancy via weighted gray edge. It dispenses dissimilar mass to diverse boundaries according to the information of boundaries called weighted grey edge [24]:

$$
\left(\int\left|w\left(f_{t}\right)^{K} f_{t, D}(D)\right|^{p} d D\right)^{\frac{1}{p}}=K i_{f}
$$

Where $w\left(f_{t}\right)$ denotes mass purpose, $\mathrm{K}$ remains constant to impose the mass of the boundaries, $f(D)$ stays on input, $i_{f}$ denotes final-illuminant to make proper color. This modifies colors as close as original colors, considerably improving ending outcome. Numerous scheme incorporates towards accurately estimating color, an appropriate weighting system must impose appropriate data about color and ignore unrelated data. Given that cue intended for estimating color, an obvious choice towards computing weight using specular edge detection methods.

\subsection{Different boundary methods}

A variety of boundary type as material, shadow or shading, specular and inter-reflection boundaries. i) Material boundaries are conversion among two dissimilar surface or else items. ii) Shading boundaries are conversion that reasons the geometry of an item, intended for an alteration in surface direction by means of light. It is also cause obstruct in the beam source. Obstruct intensity gradient, however occasionally introduce faint color gradient. iii) Inter-reflection is the cause of radiance show as of one surface against a second surface. Thus, further transform of entire lighting towards inmost near next surface, therefore color, perceived via subtracting the derivative of invariant as well as true image. The projected scheme includes various photometric descriptions. Further exclusively, quasi invariants use many flexible weighting systems, resultant within a well-designed weighting system. Quasi invariants calculate the derivative of a representation, with three photometric variants

$$
f_{D}=\left(f_{R, D}, f_{G, D}, f_{B, D}\right)^{T}
$$

Besides eliminating inconsistency, a set of derivatives making called quasi invariants. Using quasi invariants portray, three dissimilar weighting schemes.

\subsubsection{Specular boundary system}

The quasi invariants decay picture into three paths. Ridge of derived term resting on luminosity basis called specular variant which definite as 


$$
O_{D}=\left(f_{D} \cdot \stackrel{\wedge}{C}\right){ }^{\wedge} \stackrel{i}{C}
$$

where $\stackrel{\wedge_{i}}{C}=\frac{1}{\sqrt{3}}(1,1,1)^{T}$ denotes color source and dot signify vector product. The derivative reasons with places of interest and subtracted with variation as of its derivative termed as

$$
O_{D}^{t}=f_{D}-o_{D}
$$

It merely enclosed shadow shade with material boundaries and is not sensitive towards emphasizing boundaries. While the entire derivative power enclosed in three various paths, relation among specular variation vs whole sum of energy be a suggestion that a boundary is specular or else not. The specular weighting system as:

$$
W_{S}\left(f_{D}\right)=\frac{\left|o_{D}\right|}{\left\|f_{D}\right\|}
$$

Where $\left|o_{D}\right|$ describes complete rate with $o_{D}$ in addition to $\left\|f_{D}\right\|=\sqrt{f_{R, D}^{2}+f_{G, D}^{2}+f_{B, D}^{2}}$.

\subsubsection{Shadow boundary system}

By means of similar analysis happening, the shadow-shading route an invariant in addition to quasi invariant attained as:

$$
\begin{aligned}
& S_{D}=\left(f_{D} \cdot \hat{e}\right) \hat{e} \\
& S_{D}^{t}=f_{D}-S_{D}
\end{aligned}
$$

Where $\hat{e}=\frac{1}{\sqrt{R^{2}+G^{2}+B^{2}}}(R, G, B)^{T}$. This quasi-invariant is not sensitive towards shadow boundaries. Translating this yields the next effect:

$$
W_{S D}(e)=\frac{\left|S_{D}\right|}{\left\|f_{D}\right\|}
$$

\subsubsection{Material boundary system}

At last, shadow system along with quasi invariant creates extrapolate derivative taking place hue path:

$$
\begin{aligned}
& H_{D}=\left(f_{D} \cdot \hat{b}\right) \hat{b} \\
& H_{D}^{t}=f_{D}-H_{D}
\end{aligned}
$$

where denotes hue path, perpendicular on the way to preceding paths:

$$
\hat{b}=\frac{\hat{e \times} \hat{C}^{\wedge}}{|\hat{e} \times \hat{C}|}
$$

where $H_{D}^{t}$ do not enfold specular or else shadow boundaries, which then employ better weights to objects boundaries resembling

$$
W_{M}\left(f_{D}\right)=\frac{\left|H_{D}^{t}\right|}{\left\|f_{D}\right\|}
$$

To assess the control of boundary -kind classifier on the color constancy outcome. Out of these three methods specular edge type used to estimate final illuminant.

\subsection{Iterative Weighted Gray Edge}

The projected scheme initially correct deblurred imagery by an approximated illuminant. Then, last color corrected output image is given as

$$
f_{f}=f_{D}\left(\frac{1}{i_{f}}\right)^{D}
$$

Subsequently, in suggested method, a novel weighting scheme is to compute color corrected imagery for every iteration. For clearness, we will not change the weighting scheme throughout the iterations. Additionally, the early light approximate white basis $(1,1,1)^{T}$ otherwise end the color adjusted result. At last, convergence defined for predestined several iterations.

\section{Experiment Results}

Experiments results focussed towards removing blur and enhancing color. The improved underneath picture illustrates rigorous color alteration; however not succeed to improve colors completely. Deblurring method in Figure. 3 gives deblurred picture; furthermore Figure 4 yield enhanced result to improve the color. Therefore, the projected scheme yields improved performance than the conventional system via four major branches: (A) Underwater Deblurring Evaluation (B) Underwater Enhancement Evaluation, and (C) Quantitative Evaluation. These parts depict individual performances of each module.

\subsection{Underwater Deblurring Evaluation}

It is an effective scheme for reconstructing original representation. Estimated PSF is added with original to make blurry which then added with Gaussian noise to get a blurry representation, which subjected through iterative deblurring schemes to recover novel imagery. Our algorithm in Figure 3 applied to only luminance element because human vision is more responsive to luminance variation.

\subsection{Underwater Enhancement Evaluation}

The undersea turbidity removal imagery improves the eminence although it produces severe color deformation owing towards absorbing band with underneath element. Thus inaccurate statement is based on every hue band is evenly absorbed in underneath. Thus projected technique in Figure 4 employ weighted gray edge process towards make even every RGB means and thus resolve distortion problem captured in underneath. Assessment among preceding technique exposed in Figure 5. While our method can proficiently get away blur with color deformation to get proper color exclusive by artifacts and gamma adjustment to correct global contrast moreover undersea imagery tend to emerge too light. The connection among true, deblurred as well as color output illustrated in Figure 6. 


\subsection{Quantitative Evaluation}

Color adjustment using final illuminant gives final output, used by computer vision systems in many applications. Then projected algorithms give improved results as evaluated to existing scheme [30]. Table summarize metrics using Image quality Assessment and unique Image quality Assessment (SIQA). Thus projected scheme be superior than conventional scheme gives improved result.

\section{a) Ordinary IQA}

It mainly assesses contrast and structural adjustment. The most commonly used IQAs introduced as follows:Mean: Mean is the sum of all values in matrix.

$$
\mu=\frac{1}{M N} \sum_{m=0}^{M-1} \sum_{n=0}^{N-1} x_{m, n}
$$

b) Standard deviation $(S D)$ : It reflects the degree of dispersion within picture on its standard significance, and contrast in certain range. The larger SD, better the visual effect will be:

$$
S D=\sum_{m=1}^{M} \sum_{n=1}^{N} \sqrt{\frac{(f(m, n)-\mu)^{2}}{M N}}
$$

where $M$ with $N$ denotes row, column of imagery; $f(i, j)$ denoted intensity of pel moreover $\mu$ belongs to standard rate of whole image.

c) Entropy $(E)$ : An image taken as a source of random output sets $\left\{a_{i}\right\}$ and the probability with $a_{i}$ is $P\left(a_{i}\right)$ then standard amount by data in image as shown as

$$
H=-\sum_{i=1}^{L} P\left(a_{i}\right) \log _{2} P\left(a_{i}\right)
$$

Higher value of $\mathrm{E}$, more the information in picture.

d) Mean squared error (MSE): The full-reference excellence metric computed via average squared intensity differences of distortion along with reference representation pels as

$$
M S E=\frac{1}{M N} \sum_{m=1}^{M} \sum_{n=1}^{N}\left[f(m, n)-f^{\prime}(m, n)\right]
$$

where $M$ and $N$ denotes row and column of the imagery, $f(m, n)$ remains original with $f^{\prime}(m, n)$ denotes deblurred picture.

e) Peak SNR (PSNR): It is used as index for signal distortion. Larger the PSNR smaller the distortion and expressed as:

$$
P S N R=10 \log \frac{f_{\max }^{2}}{M S E}
$$

where $f_{\max }$ stays on largest gray value, in general $f_{\max }=255$.

f) Structural similarity (SSIM): Generally, the ocular view is extremely modified to extract data on picture, thus measures restored picture excellence using three mechanism specifically; luminance $L(D, E)$, contrast $c(D, E)$ along with structure comparison $S(D, E)$. All these combined to give up whole similarity computation as:

$$
S(m, n)=F(L(D, E), c(D, E), S(D, E))
$$

The similarity of the two images is dependent on SSIM, and has a value between $[0,1]$. When the value is close to 1 , the two images are more similar. SD reflects dissimilarity of the image; E reflects the data; later MSE, PSNR and SSIM reflects the degree of distortion. Higher MSE, lower PSNR and SSIM scores imply greater dissimilarity among enhanced results and referenced deblurred image. This measures often used for simple calculation enclose clear and suitable for optimization. Conversely, these approaches cannot be simply adopted, because preceding IQA metrics are usually unsuitable for relevance intend to measure distortion intensity rather than visibility in imagery.

\subsubsection{Special IQA}

Some IQAs designed particularly for image from different views as follows.

\section{a) Image Visibility Measurement (IVM)}

Inspired with blind assessment indicator, an extra picture visibility measurement employ perceptible boundary segmentation as

$$
I V M=\frac{n_{v}}{n_{\text {total }}} \log \sum_{D \in \mathfrak{I}} C(D)
$$

where $n_{v}$ represents the amount of perceptible boundaries, $n_{\text {total }}$ denotes amount of boundaries, $C(D)$ remains average dissimilarity, along with $\mathfrak{s}$ denotes picture region with perceptible boundary.

\section{b) Histogram correlation coefficient (HCC)}

A good deblurring technique should allow improved picture towards Histogram allocation. It employs dual color imagery as standard to measure the act of color enhancement.

\section{c) Contrast gain}

The dissimilarity of clear picture is much higher than that of deblurred imagery, that check dissimilar enhancing system. Higher contrast, better the scheme to be. Global dissimilarity is for evaluating different techniques. It signifies mean contrast comparison among enhanced as well as deblurred image by

$$
C_{\text {gain }}=\bar{C}_{J}-\bar{C}_{I}
$$

where $\bar{C}_{J}$ in addition to $\bar{C}_{I}$ represents mean contrast of enhanced and deblurred representation, respectively.

\section{d) Ocular dissimilarity assess (ODS)}

It compute the amount of perception using

$$
O D S=100 * R_{v} / R_{t}
$$

where $R_{v}$ represents amount with limited region, SD is higher than known boundary with $R_{t}$ remains entire amount of limited region. We chose OTSU image segmentation algorithm that adaptively compute the limit. It uses local standard deviation that denotes dissimilarity picture towards determining visibility. 
A.Chrispin Jiji et al. / Advances in Science, Technology and Engineering Systems Journal Vol. 3, No. 6, $97-104$ (2018

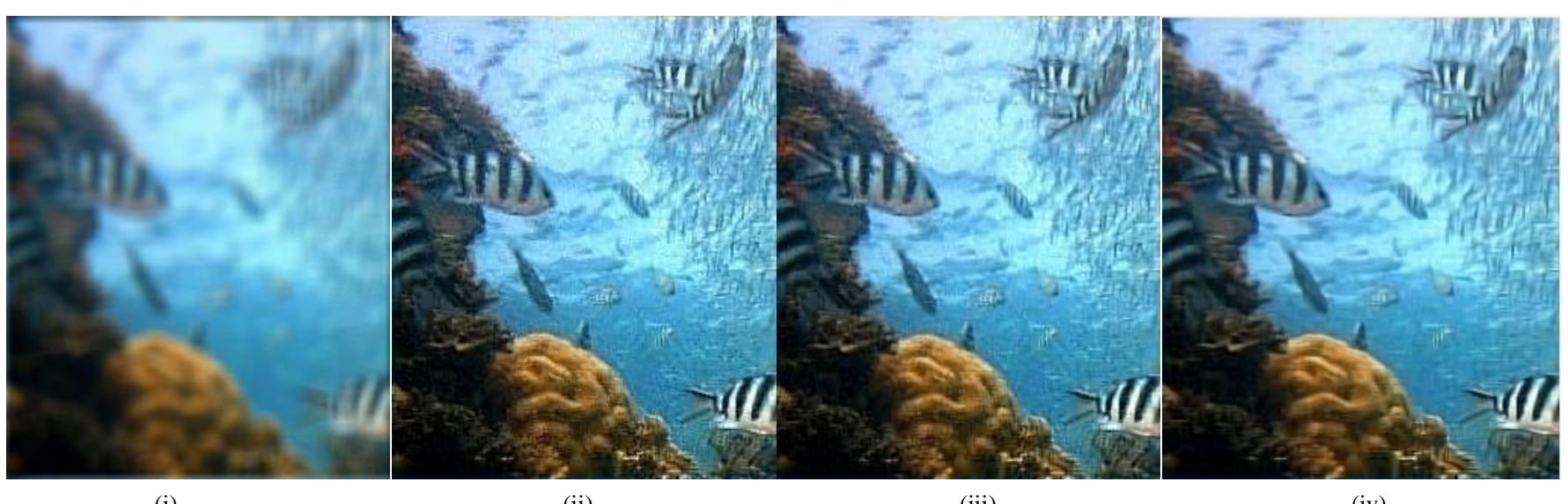

(i)

(ii)

(iii)

(iv)

Figure 3: Underwater Turbidity Removal. (i)Blurred picture (ii) ASDS (iii) ASDS using AR (iv) ASDS using AR and NL

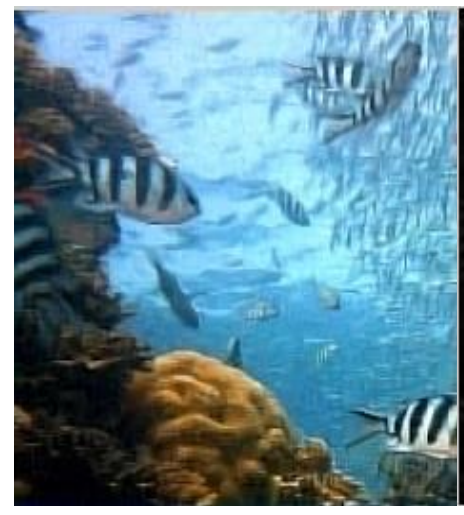

a) Deblurred

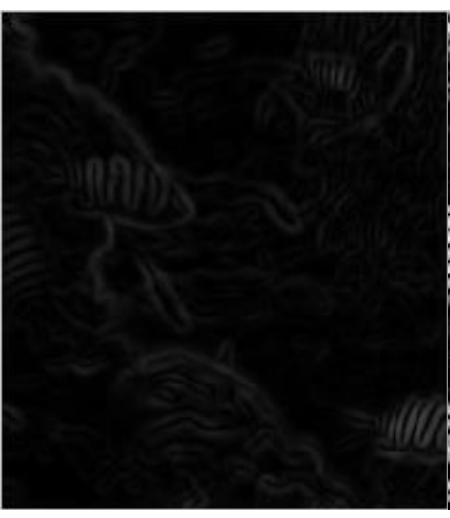

b) grad_im

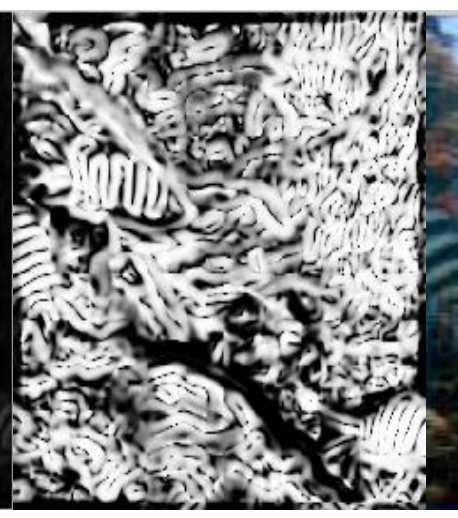

c) weight_map

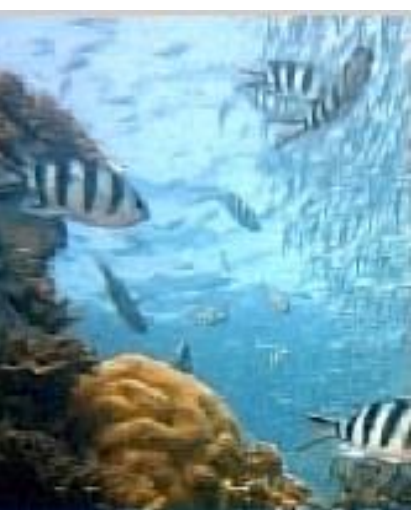

d) Ours

Figure 4: Proposed Method

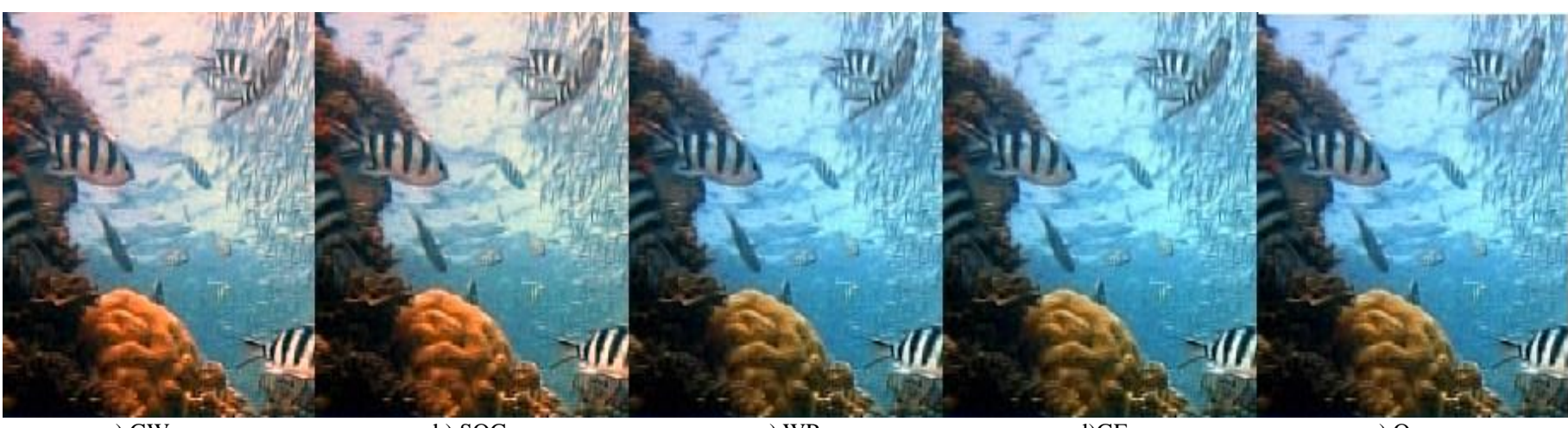

a) GW

b) SOG

c) WP

d)GE

e) Ours

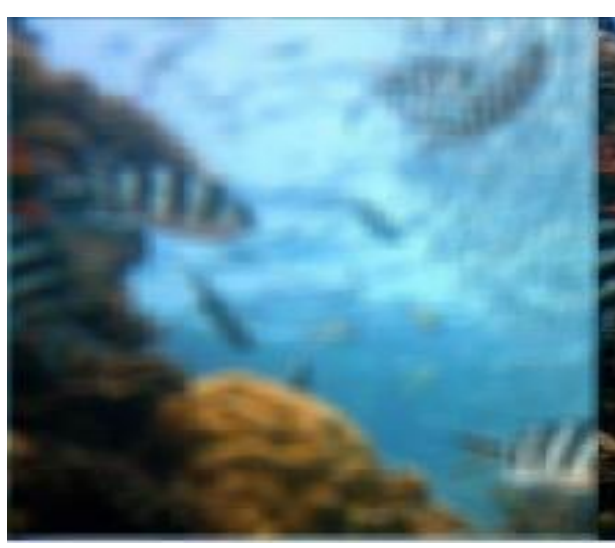

a) Blurred Image

Figure 5: Assessment among preceding technique

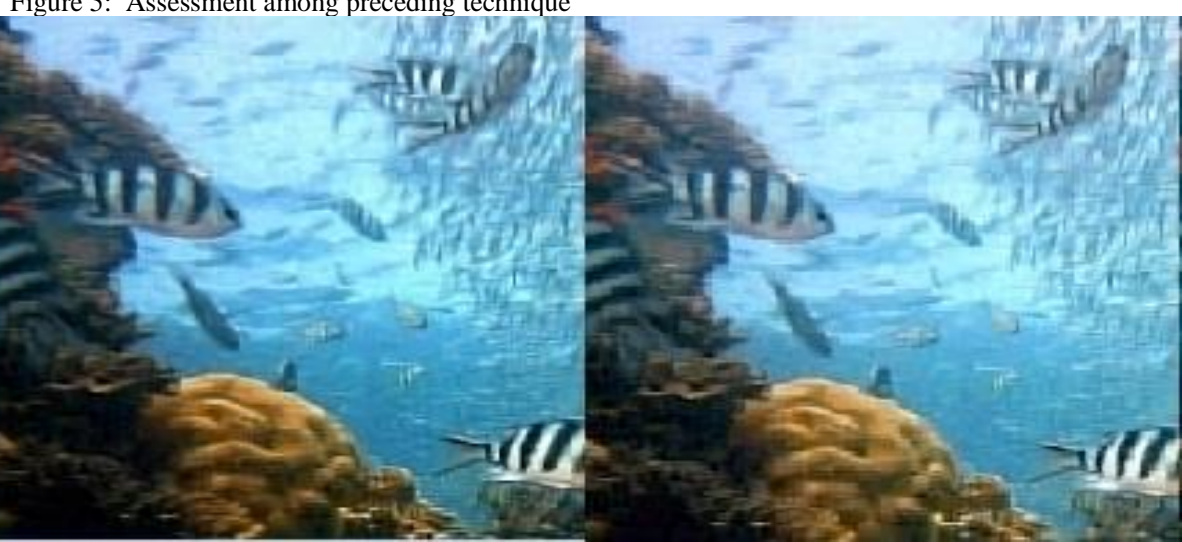

b) Deblurred

Figure 6: Input and output imagery c) Ours 
A.Chrispin Jiji et al. / Advances in Science, Technology and Engineering Systems Journal Vol. 3, No. 6, 97-104 (2018

Table 1: Estimation of eminence metrics of Image quality Assessment (IQA) and Special Image quality Assessment (SIQA) of Proposed Method

\begin{tabular}{|l|l|l|l|l|l|l|l|l|l|l|l|}
\hline & Methods & M & SD & E & PSNR & RMSE & SSIM & ODS & HCC & CG & UQI \\
\hline \multirow{5}{*}{ ID } & ASDS & 127.3 & 76.98 & 7.911 & 25.31 & 19.916 & 0.694 & 67.867 & 0.444 & 0.465 & 0.948 \\
\cline { 2 - 13 } & ASDSAR & 127.31 & 76.834 & 7.919 & 25.73 & 19.117 & 0.716 & 64.82 & 0.475 & 0.422 & 0.962 \\
\cline { 2 - 13 } & ASDSARNL & 127.34 & 76.66 & 7.933 & 26.16 & 17.816 & 0.742 & 58.449 & 0.531 & 0.334 & 0.940 \\
\hline \multirow{4}{*}{ CA } & GW & 129.6 & 72.443 & 7.798 & 65.038 & 0.142 & 0.9983 & 62.604 & 0.070 & 0.054 & 0.976 \\
\cline { 2 - 13 } & SOG & 128.73 & 72.057 & 7.794 & 65.745 & 0.132 & 0.9986 & 62.05 & 0.096 & 0.051 & 0.976 \\
\cline { 2 - 13 } & WP & 127.15 & 76.134 & 7.923 & 69.992 & 0.081 & 0.9999 & 58.449 & 0.477 & 0.045 & 0.981 \\
\cline { 2 - 13 } & GE & 127.55 & 76.954 & 7.931 & 69.993 & 0.081 & 0.9999 & 58.449 & 0.978 & 0.044 & 0.981 \\
\cline { 2 - 12 } & Ours & 127.42 & 76.812 & 7.910 & $\mathbf{7 0 . 0 0 2}$ & $\mathbf{0 . 0 8 1}$ & $\mathbf{0 . 9 9 9 9}$ & 58.449 & 0.962 & 0.044 & $\mathbf{0 . 9 8 1}$ \\
\hline
\end{tabular}

Generally, higher ODS, clearer the improved picture.

\section{e) Universal quality index (UQI)}

It mainly assess performance of UQI among original as well as the improved imagery given as

$$
Q=\frac{4 \sigma_{d e} \bar{d} \bar{e}}{\left(\sigma_{d}^{2}+\sigma_{e}^{2}\right)\left[(\bar{d})^{2}+(\bar{e})^{2}\right]}
$$

Where

$$
\begin{aligned}
& \bar{d}=\frac{1}{N} \sum_{m=1}^{N} d_{m}, \quad \bar{e}=\frac{1}{N} \sum_{m=1}^{N} e_{m} \\
& \sigma_{d}^{2}=\frac{1}{N-1} \sum_{m=1}^{N}\left(d_{i}-\bar{d}\right)^{2}, \quad \sigma_{e}^{2}=\frac{1}{N-1} \sum_{m=1}^{N}\left(e_{m}-\bar{e}\right)^{2} \\
& \sigma_{d e}=\frac{1}{N-1} \sum_{m=1}^{N}\left(d_{m}-\bar{d}\right)\left(e_{m}-\bar{e}\right)
\end{aligned}
$$

Traditional UQI criterion both uses a picture with high excellence as the reference image. Thus, higher UQI, better the compared image. However, the deblurred imagery is always chosen as of reference imagery, so large UQI do not means that the imagery is of high excellence. The improved representation with best visibility may have smallest UQI.

\section{Conclusion}

We projected an effective technique namely an underwater turbidity removal scheme with adaptive sparse domain selection (ASDS) methodology significantly improves undersea imagery, and consequently, results of image deblurring. If the deblurred imagery has any lighting problem, then; we used color corrected method using Weighted Gray Edge to find the clear image.

The experimental results shown that the projected means illustrate towards removing turbidity outperforms several stateof-the-art restoration scheme in excellence metrics along with ocular excellence. Finally, we use a color correction scheme to clear the lighting (color) in state-of-the-art images.

\section{Acknowledgment}

The authors thank the reviewers for their thorough and helpful remarks.

\section{References}

[1]. M. Kocak, Fracer Dal, M, F Cai and Sche, "A focus on recent development and trends in Underwater Imagng," Mare Tech. Socie. Jorn, Mar-2008.

[2]. Gian. Lu. F, "Visual inspection of Sea Bottom Structures by an Autonomous Underwater Vehicle," IEEE Transac Systm., Man, Cybern. Oct-2001.
[3]. Alberto O, Miqel S, and Gab.O, "A Vision System for an Underwater Cable Tracker," Machn. Vision. Appln., 2002.

[4]. Adria. O and Eman. T, "Detecting Man-Made Objects in Unconstrained Subsea Videos," Prod. BMV-2002,

[5]. BA. Level and L. Berg, "Control of Underwater Vehicles in full unsteady flow," IEEE Jrn. Oceac. Engg-2009.

[6]. CH. Maze, "In Situ Measurement of Reflectance and Fluorescence spectra to support Hyperspectral Remote Sensing and Marine Biology Research," IEEE oceanc-2006.

[7]. Yaac. K and Jeffrey R, "Analysis of Hull remains of the Dor D vessel," Intn. Jrn. of Nautial Archeo- 2001.

[8]. BL. McGla, "A Computer model for Underwater Camera System," Proc of spie 1979.

[9]. J. Jaf, "Computer Modeling and Design of Optimal Underwater Imaging Systems," IEEE Journ of Oceanic Engi -1990.

[10]. J. Fun, B. Brya, and J. Heck, "Handbook of Underwater Imaging System Design," TP303 - 1972.

[11]. Timo H, Thom P, F. Chap, and Rob. CT, “A Range-Gated Laser System for Ocean Floor Imaging," Marin Tech So Jo-1983.

[12]. John W and Kenn. V, "Point spread functions in Ocean Water: Comparison between Theory and Experiment," Appd Opti-1991.

[13]. Tor M and Gre Du, "A statistical learning based method for color correction of Underwater Image", Advn artifi intelli theory-2005

[14]. Chike and Fu"Automatic white balance for digital still camera," Inf.orm Scien Jrnl- 2006

[15]. Weng, Ho C, and Fu, "A Novel automatic white balance method for digital still Cameras," IEEE_ Inter .Sym Ckts \& Sys- 2015.

[16]. EY La, "Combining grey world \& Retinex theory for automatic White Balance in digital photography,” Inter Sym Consum Elec, 2005.

[17]. Xial. W, Xiang. Z and Jia.W, "Model-Guided Adaptive Recovery of Compressive Sensing," Proc Data Compre Confe, - 2009.

[18]. Juli. M, Franc. B, Jean. P, Guil. S and Andre. Zn, "Non-Local sparse models for Image restoration," Intern Conf $r$ on Compt Visn-2009.

[19]. Stef. K, Stanley. O, and Peter. W. J, "Deblurring and Denoising of images by Non-Local Functional," Multiscl Modl and Simun- 2005.

[20]. Mattan. P, Michael. E, Hiro. T, and Paymn. M, "Generalizing the NonlocalMeans to Superresolution Reconstruction," IEEE Transac. Img Pros- 2009.

[21]. Riz, Gat and Mar "Color Correction between Gray World and White Patch," Humn vis \& Elecr,2002

[22]. Finly and Trez "Shades of grey and colour constancy," Clr \& Imag Confr, 2004.

[23]. J Van, T Geve "Edge based colour constancy," proc.IEEE Trans on Img Proces, 2010

[24]. Gijs, T Gevrs, and Van, "Improving Color constancy by photometric edge weighting," proc. IEEE_Trans. on Patrn Analy \& Machine Intelli-2012.

[25]. A.Chrispin Jiji,Vivek M "Underwater Turbidity Removal through 1ll-posed optimization of sparse modeling," ICPCSI (2017).

[26]. Weish D, Lei Zhg, Gua Sh, Xiaon Wu, "Image Deblurring and Super resolution by Adaptive Sparse Domain Selection and Adaptive Regularization", IEEE Transc on Imge Proc- 2011.

[27]. We Dong, Le Zhang, Guan Shi, Xiao W, "Image Reconstruction with Locally Adaptive Sparsity and Nonlocal Robust Regularization", Sigl Proces: Img Commn -2011.

[28]. Mauo Delb, Pu Mu , Andr Alman "Non -Parametric Sub-Pixel local point spread function estimation," Img Proces-2012.

[29]. Yoav. S and Nir. K, "Recovery of Underwater Visibility and Structure by Polarization Analysis," IEEE Jol of Ocea Engg, 2005.

[30]. Y Xu, , J Wen, L Fei1, and Zheng Z, "Review of Video and Image Defogging algorithms and related studies on Image Restoration and Enhancement", IEEE open acces Jorn-2016. 